\title{
Breast Sclerosing Adenosis
}

National Cancer Institute

\section{Source}

National Cancer Institute. Breast Sclerosing Adenosis. NCI Thesaurus. Code C5205.

Breast adenosis characterized by the proliferation of acini, a lobulated architectural pattern, and stromal sclerosis. The luminal epithelial and myopepithelial cells are preserved. Microcalcifications and foci of apocrine metaplasia may be present. 